\title{
Identification of univalent chromosomes in monosomic lines of the Gossypium hirsutum L. cotton type with the help of cytogenetic markers
}

\author{
M.F. Sanamyan*, Sh.U. Bobokhujayev \\ National University of Uzbekistan named after M. Ulugbek, Tashkent, Uzbekistan
}

DOI 10.18699/ICG-PlantGen2019-81

(c) Autors, 2019

* e-mail: sanam_marina@rambler.ru

\begin{abstract}
The lack of clear morphological markers of cotton chromosomes contributed to the development of an unconventional method for marking chromosomes using translocations. Cytogenetic identification and numbering of univalent chromosomes in 25 monosomic lines of the NUU cytogenetic collection using a set of test lines with identified chromosomes made it possible to establish that four monosomic lines have univalent of chromosome 2; 15 lines, of chromosome 4; four lines, of chromosome 6; one line, of Atsubgenome chromosome 7; and one line, of cotton Dt-subgenome chromosome 18 . The remaining 21 lines were duplicates of the three non-homologous chromosomes.
\end{abstract}

Key words: cotton (G. hirsutum L.); translocation lines; identification of univalent chromosomes.

\section{Introduction}

Works on the creation of translocation tester sets, which were obtained from five plant species - maize, barley, pea, rye, and tomato - are widespread. M.S. Brown et al. (1980) obtained 62 G. hirsutum translocation lines using X-, $\gamma$, Bikini radiation and irradiation of seeds or pollen of various varieties with fast neutrons, as well as several lines. In 58 of these lines, two nonhomologous chromosomes were involved; in three, three chromosomes in each; and in one, four. For identification and numbering of chromosomes, studies were conducted on the assignment of translocated chromosomes to subgenomes. As a result of the identification, it turned out that chromosome 26 was not involved in any of the translocations and was determined by the exclusion method.

For many years, the National University of Uzbekistan has been conducting studies on the induction of cotton plants with various chromosomal aberrations using various methods of induced mutagenesis. As a result, a unique cotton cytogenetic collection was created, including monosomic, monotelodisomic and translocation lines (Sanamyan et al., 2014). This article discusses the unified identification of univalent chromosomes in monosomic cotton lines using a well-defined tester set of cotton translocation lines with identified chromosomes.

\section{Materials and methods}

Hybrid monosomic cotton plants obtained from crossing monosomic lines of the cytogenetic collection of cotton G. hirsutum L. of the National University of Uzbekistan (NUUz) with a well-defined tester set of cotton translocation lines from the Cotton Cytogenetic Collection at Texas A\&M AgriLife Research served as material for research. Cytogenetic translocation markers were kindly provided by Professor David Stelly through the ARS-USDA exchange program. In the process of cytological analyses, meiosis was studied at the stage of metaphase 1 (MI) in pollen mother cells (PMC).

\section{Results and discussion}

As a result of cytogenetic analysis of hybrids obtained from crossing monosomic cotton lines with the tester set of translocation lines with identified chromosomes, it turned out that in the case of crosses with six translocation lines - TT 2L-6R, TT 2L-3Lb, TT 2R-3La, TT 2R-8Ra, TT 2R-8Rb and TT 2R$14 \mathrm{R}$ - homology of univalent chromosomes was established in four monosomic lines (Mo11, Mo16, Mo19 and Mo93) and one of the translocated chromosomes in the above translocations, since 24 bivalent plus one trivalent translocations in MI meiosis were observed (Figure 1,a). In the tester line TT2L-6R, chromosomes 2 and 6 are involved in translocation; in the TT2L-3Lb and TT2R-3La lines, chromosomes 2 and 3 are involved in translocation; in the TT2R-8Ra and TT 2R$8 \mathrm{Rb}$ lines, chromosomes 2 are involved in translocation with chromosome 8; and in the TT2R-14R line, chromosomes 2 and 14 are involved in translocation. Therefore, one of these chromosomes is homologous to the univalent chromosome in the monosomic lines Mo11, Mo16, Mo19 and Mo93. Since in six translocation lines one common chromosome 2 is involved, the univalent chromosomes in the monosomic lines Mo11, Mo16, Mo19 and Mo93 are chromosome 2 of the At subgenome of cotton, and these monosomic lines are duplicates. In the analysis of hybrids obtained from crosses of seven cotton monosomic lines (Mo31, Mo70, Mo72, Mo73, Mo75, Mo76, Mo89) with two tester translocation lines, TT4L-19R and TT4R-15L, the homology of the univalents of these seven lines with one of the translocated chromosomes was established since monosomic translocation hybrids in MI meiosis showed 24 bivalents plus one trivalent.

In the tester line TT4L-19R, chromosomes 4 and 19 are involved in translocation, and in TT4R-15L, chromosomes 4 and 15 are involved in translocation, therefore, one of these three chromosomes is homologous to the univalent chromosome in the monosomic lines Mo31, Mo70, Mo72, Mo73, Mo75, Mo76, and Mo89. Since one common chromosome 4 

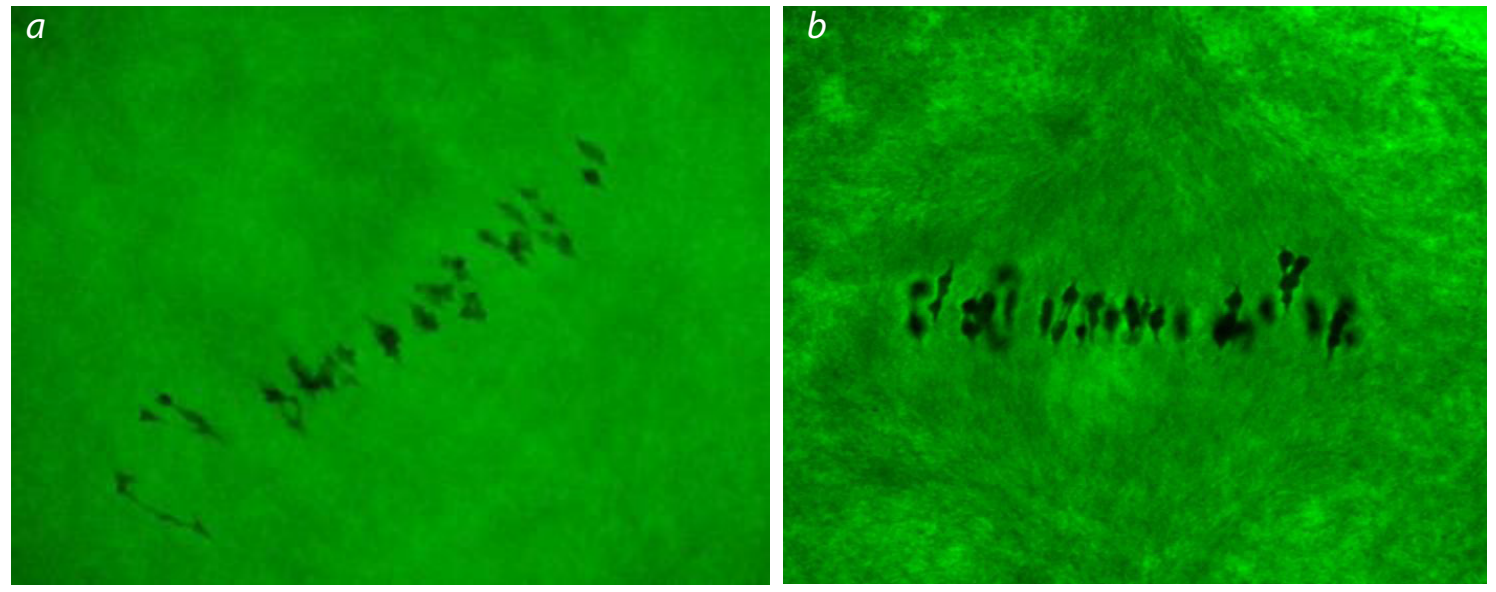

Figure 1. "Critical configurations" of the chromosomes at meiotic metaphase 1 , showing 24 bivalents and 1 trivalent in cotton $\mathrm{F}_{1}$ hybrid monosomic plants from the crosses: $a-$ Mo16 ×TT2R-8Rb; $b$ - Mo58 × TT4L-19R (24" + 1"').

is involved in both translocation lines, the univalent chromosomes of the monosomic lines Mo31, Mo70, Mo72, Mo73, Mo75, Mo76, Mo89 are cotton At-subgenome chromosome 4, and these monosomic lines are duplicates.

The study of hybrids of the other eight monosomic lines of cotton (Mo7, Mo38, Mo58, Mo59, Mo60, Mo69, Mo71 and Mo81) was carried out with only one of two translocation lines, TT4L-19R or TT4R-15L. As a result, the homology of the univalents of these eight lines with one of the translocated chromosomes was established, since monosomic translocation hybrids in MI meiosis showed 24 bivalents plus one trivalent (Figure 1, b). Since chromosomes 4 and 19 are involved in translocation in the tester line TT4L-19R, and chromosomes 4 and 15 are involved in translocation in the TT4R-15L line, therefore, one of these three chromosomes is homologous to the monosomics Mo7, Mo38, Mo58, Mo59, Mo60, Mo69, Mo71 and Mo81. Since one common chromosome 4 is involved in both translocation lines, it can be assumed that the univalent chromosomes in Mo7, Mo38, Mo58, Mo59, Mo60, Mo69, Mo71 and Mo81 are chromosome 4 of the cotton Atsubgenome, and these monosomic lines are duplicates. The chromosomal locations of chromosome-specific SSR markers on $\mathrm{F}_{1}$ hybrids with the participation of lines Mo7, Mo38, Mo58, Mo59, Mo60, Mo69, Mo71 and Mo81 confirmed these data (Sanamyan et al., 2016).

In the study of four monosomic cotton lines (Mo13, Mo34, Mo67, Mo95) using four translocation lines, TT 3L-6L, TT 6L7L, TT 6L-10R, and TT 6L-14L, the homology of the univalent chromosomes of these four monosomic lines was established and one of the translocated chromosomes in the above-listed translocations, since 24 bivalents plus one trivalent were observed in monosomic translocation hybrids in MI. Since one common chromosome 6 participates in four translocation lines, the univalent chromosomes of the monosomic lines Mo13, Mo34, Mo67, Mo95 are chromosome 6 of the cotton At subgenome, and these monosomic lines are duplicates.

The study of the monosomic line Mo27 in four crosses, Mo27×TT 1L-7L, Mo27×TT 7L-12R, Mo27×TT 7R-11R and Mo27 $\times$ TT 7R-21R, revealed univalent chromosome and one of the translocated chromosomes, because monosomic hybrids in MI showed pairing chromosomes in the form of 24 bivalents and one trivalent. Since one common chromosome 7 is involved in the four translocation lines, the univalent chromosome in the monosomic line Mo27 is cotton At-subgenome chromosome 7.

In the study of the monosomic line Mo 48 in one variant of crossing with the translocation line TT7L-18R in the MI of meiosis, 24 bivalents plus one trivalent were found, which indicated the homology of the univalent chromosome in Mo48 and one of translocated chromosomes in translocation line TT7L-18R. Because in the tester line TT7L-18R, chromosomes 7 and 18 are involved in translocation, the univalent chromosome in the monosomic line Mo48 is homologous to one of the two chromosomes. Unfortunately, there is no second translocation line in the tester set of lines with identified chromosomes that involved chromosome 18 . Therefore, to determine to which of the two chromosomes of this translocation line the univalent chromosome of Mo48 is homologous, molecular genetic analysis of the monosomic interspecific $\mathrm{F}_{1}$ hybrid (Mo48 $\times$ Pima3-79) was used, which showed the presence of only a $G$. barbadense-specific SSR marker band (BNL3280) and the absence of the respective G. hirsutum allele (Sanamyan et al., 2016). Because this SSR marker had previously been assigned to chromosome 18 of the Dt subgenome of cotton, we can assume that the monosomic line Mo48 of the NUU collection has a monosomy on chromosome 18 of the Dt subgenome.

\section{Conclusion}

The use of translocation lines with identified chromosomes allowed us to bring the numbering of univalent chromosomes in the monosomic lines of our collection to conformity with the generally accepted nomenclature. Cytogenetic identification and numbering of univalent chromosomes in 25 monosomic lines of the cytogenetic collection of NUU allowed us to establish that four monosomic lines have univalent of chromosome 2; 15 lines, of chromosome 4; four lines, of chromosome 6; one line, of At-subgenome chromosome 7; and one line, of cotton Dt-subgenome chromosome 18. The overwhelming majority of monosomic lines were detected 
by the most frequently registered cotton monosomics - by chromosomes 2,4 and 6.

A comparative analysis of the first 20 identified monosomics of cotton obtained in the USA revealed similar trends, since the study revealed 7 monosomics on chromosome 2 , seven on chromosome 4 , three on chromosome 6 and one on chromosomes 1, 17 and 18 (Brown, Endrizzi, 1964). The similarity of the data obtained in the study of different collections indicates that, despite the differences in genotypic environments and methods for producing monosomics, cotton has a surprising coincidence of data on a higher frequency of monosomics appearing precisely on chromosomes 2, 4 and 6, while monosomics on other chromosomes of the set appear at a much lower frequency, and eight non-homologous chromosomes (At-subgenome 5, 8, 13 and cotton Dt-subgenome 14, 15, 19, 22 and 24) have never been detected (Saha et al., 2012). Apparently, the centromeric regions of certain chromosomes are subject to more frequent breakdown events and the genome as a whole remains tolerant to the loss of large At-subgenome chromosomes without much effect on viability and fertility, while the chromosomes of some small Dt-subgenome chromosomes are not subject to any changes due to incompatibility with vitality.

\section{References}

Brown M.S., Endrizzi J.E. The origin, fertility and transmission of monosomic in Gossypium. Amer. J. Bot. 1964;51(1):108-115.

Brown M.S. Identification of the chromosomes of Gossypium hirsutum L. by means of translocations. J. Hered. 1980;71(4):266-274.

Saha S., Stelly D.M., Raska D.A., Wu J., Jenkins J.N., McCarty J.C., Makamov A. Chapter 6. Chromosome substitutions lines: concept, development and utilization in the genetic improvement of upland cotton. In: Abdurakhmonov I.Y. (Ed). Plant Breeding. In Tech: Croatia, 2012;107-128.

Sanamyan M.F., Petlyakova J., Rakhmatullina E.M., Sharipova E. Chapter 10. The Cytogenetic Collection of Uzbekistan. In: Abdurakhmonov I.Y. (Ed). World Cotton Germplasm Resources. In Tech: Croatia, 2014;247-287.

Sanamyan M.F., Makamov A.K., Bobokhujaev Sh.U., Usmonov D.E., Buriev Z.T., Saha S., Stelly D.M. Chapter 8. The utilization of the translocation lines and microsatellite markers for the identification of unknown cotton monosomic lines. In: Abdurakhmonov I.Y. (Ed). Cotton Research. In Tech: Croatia, 2016;167-183.

Acknowledments. This work was supported by the Committee on Science and Technology and the Ministry of Innovations of the Republic of Uzbekistan (grants F-5-31 and OT-A-KH-2018-379).

Conflict of interest. The authors declare no conflict of interest. 